\title{
Clinical and CT imaging features of the COVID-19 pneumonia: Focus on pregnant women and children
}

\author{
Huanhuan Liu ${ }^{\mathrm{a}, 1}$, Fang Liu ${ }^{\mathrm{b}, 1}$, Jinning $\mathrm{Li}^{\mathrm{a}}{ }^{\mathrm{a}}$, Tingting Zhang ${ }^{\mathrm{a}}$, Dengbin Wang ${ }^{\mathrm{a}, *}$, \\ Weishun Lan ${ }^{\mathrm{b}, *}$ \\ a Department of Radiology, Xinhua Hospital, Shanghai Jiao Tong University School of Medicine, No. 1665 Kongjiang Road, Yangpu District, Shanghai 200092, \\ China \\ ${ }^{\mathrm{b}}$ Department of Radiology, Maternal and Child Health Hospital of Hubei Province, No.745 Wuluo Road, Hongshan District, Wuhan 430070, Hubei Province, \\ China
}

\section{A R T I C L E I N F O}

\section{Article history:}

Accepted 2 March 2020

Available online $\mathrm{xxx}$

\section{Keywords:}

COVID-19 pneumonia

Pregnancy

Children

CT

\section{S U M M A R Y}

Background: The ongoing outbreak of COVID-19 pneumonia is globally concerning. We aimed to investigate the clinical and CT features in the pregnant women and children with this disease, which have not been well reported.

Methods: Clinical and CT data of 59 patients with COVID-19 from January 27 to February 14, 2020 were retrospectively reviewed, including 14 laboratory-confirmed non-pregnant adults, 16 laboratory-confirmed and 25 clinically-diagnosed pregnant women, and 4 laboratory-confirmed children. The clinical and CT features were analyzed and compared.

Findings: Compared with the non-pregnant adults group $(n=14)$, initial normal body temperature (9 [56\%] and 16 [64\%]), leukocytosis (8 [50\%] and 9 [36\%]) and elevated neutrophil ratio (14 [88\%] and 20 $[80 \%])$, and lymphopenia (9 [56\%] and $16[64 \%])$ were more common in the laboratory-confirmed $(n=16)$ and clinically-diagnosed $(n=25)$ pregnant groups. Totally 614 lesions were detected with predominantly peripheral and bilateral distributions in 54 (98\%) and 37 (67\%) patients, respectively. Pure ground-glass opacity (GGO) was the predominant presence in 94/131 (72\%) lesions for the non-pregnant adults. Mixed consolidation and complete consolidation were more common in the laboratory-confirmed (70/161 [43\%]) and clinically-diagnosed (153/322 [48\%]) pregnant groups than $37 / 131$ (28\%) in the non-pregnant adults $(P=0.007, P<0.001)$. GGO with reticulation was less common in $9 / 161(6 \%)$ and $16 / 322(5 \%)$ lesions for the two pregnant groups than $24 / 131(18 \%)$ for the non-pregnant adults $(P=0.001, P<0.001)$. The pulmonary involvement in children with COVID-19 was mild with a focal GGO or consolidation. Twentythree patients underwent follow-up CT, revealing progression in 9/13 (69\%) at 3 days whereas improvement in 8/10 (80\%) at 6-9 days after initial CT scans.

Interpretation: Atypical clinical findings of pregnant women with COVID-19 could increase the difficulty in initial identification. Consolidation was more common in the pregnant groups. The clinically-diagnosed cases were vulnerable to more pulmonary involvement. CT was the modality of choice for early detection, severity assessment, and timely therapeutic effects evaluation for the cases with epidemic and clinical features of COVID-19 with or without laboratory confirmation. The exposure history and clinical symptoms were more helpful for screening in children versus chest $\mathrm{CT}$.

(c) 2020 The Author(s). Published by Elsevier Ltd on behalf of The British Infection Association. This is an open access article under the CC BY-NC-ND license. (http://creativecommons.org/licenses/by-nc-nd/4.0/)

\footnotetext{
* Corresponding authors.

E-mail addresses: wangdengbin@xinhuamed.com.cn (D. Wang), lws7375@126.com (W. Lan).
}

1 These authors contributed equally to this work.

\section{Introduction}

The pneumonia of unknown etiology occurred in December, 2019 in Wuhan, Hubei Province, China, has been confirmed to be caused by a novel coronavirus. ${ }^{1-3}$ The newly identified virus has been recently named "severe acute respiratory syndrome coronavirus $2^{\prime \prime}$ (SARS-CoV-2), and the disease caused by SARSCoV-2 was designated coronavirus disease 2019 (COVID-19) by the 
World Health Organization (WHO). ${ }^{4}$ The SARS-CoV-2 is the seventh member of enveloped RNA coronavirus besides coronavirus 229E, OC43, NL63, HKU1, SARS-CoV, and Middle East respiratory syndrome coronavirus (MERS-CoV). ${ }^{1}$ The initial clusters of patients with COVID-19 pneumonia were epidemiologically linked to local seafood market in Wuhan, and person-to-person transmission was subsequently confirmed. ${ }^{5-7}$ It has rapidly spread globally. As of February 18, 72,532 cases were laboratory-confirmed and clinically-diagnosed, and 6242 were suspected in China. ${ }^{8}$ The reproductive number (R0), defined as the expected number of secondary infectious cases generated by an infectious case in a susceptible population, is estimated to $3.77 .{ }^{9}$

Recent publications have depicted the epidemiological and clinical characteristics of patients infected with SARS-CoV-2.,3,7,9-11 The definite diagnosis of COVID-19 is expected with the novel coronavirus nucleic acid positive in swabs, sputum, or secretions from the lower respiratory tract, or blood by real-time reverse transcription polymerase chain reaction assay (RT-PCR). However, the new coronavirus nucleic acid kits are in short supply and time-consuming in manipulation. Furthermore, false negatives may exist in some cases due to the insufficient viral materials in the specimen. Recent studies have reported the cases with initial SARS-CoV-2 negative but typical CT findings, ${ }^{12,13}$ however, most of them turned out to be positive after repeated tests for SARS-CoV-2 nucleic acid. Therefore, chest CT imaging plays a critical role in the surveillance and diagnosis of COVID-19 pneumonia with a higher sensitivity and efficiency than the chest X-rays do. Guan et al. also reported that $76.4 \%$ cases with viral pneumonia findings at CT were verified COVID-19. ${ }^{11}$ Based on the epidemiological characteristics of COVID-19 pneumonia, patients with typical CT imaging features in Hubei Province will be grouped as clinicallydiagnosed cases, contributing to the total number of confirmed diagnosis according to the "Diagnosis and Treatment for COVID-19 Pneumonia (Fifth Trial Version)" released by the National Health Commission (NHC) of China since February 12, 2020. ${ }^{14}$

Pregnant women are more susceptible to infectious diseases due to the immune suppression. ${ }^{15}$ Previous studies found that there was a male-dominated tendency and few cases occurred in children for COVID-19 pneumonia., ${ }^{3,9}$ To the best of our knowledge, there are few published data involving the clinical and chest CT findings of COVID-19 pneumonia in pregnant women and children. ${ }^{16,17}$ In this present study, the clinical and chest CT findings of COVID-19 pneumonia were investigated for the pregnant women and children in comparison with non-pregnant adults from two centers. The CT features were further compared between the laboratory-confirmed and clinically-diagnosed pregnant groups, which could favor the surveillance and diagnosis.

\section{Methods}

\section{Patients}

This study was approved by the Institutional Ethics Committee of relevant centers. The informed consent requirement was waived for this retrospective study. Clinical and chest CT imaging data of 60 patients with laboratory-confirmed and clinically-diagnosed COVID-19 pneumonia were reviewed. One clinically-diagnosed patient was afterwards excluded for positive mycoplasma infection. Finally, a total of 59 patients from two centers were recruited in this study, in which three non-pregnant adults were from Xinhua Hospital and the other patients from Maternal and Child Health Hospital of Hubei Province including 41 pregnant women, 11 non-pregnant adults, and 4 children. For the patients who underwent SARS-Cov-2 test, the throat swab samples were collected for RT-PCR in accordance with the WHO guideline. ${ }^{18}$

\section{CT imaging acquisition}

All the patients underwent non-enhanced chest CT examinations for detecting COVID-19 pneumonia in the supine position during end-inspiration. The CT scans of non-pregnant adults were performed with a 64-section multi-detector CT scanner (uCT780, United imaging, Shanghai, China, or Optima 660, GE Medical System, Milwaukee, WI, USA). The CT scans of pregnant women and children were all performed with the same 64-section multi-detector CT scanner (Optima 660, GE Medical System, Milwaukee, WI, USA). The protocols were as follows. For non-pregnant adults: tube voltage $120 \mathrm{kV}$, automatic tube current (120-380) $\mathrm{mA}$, thickness (5-7) $\mathrm{mm}$, slice interval $5 \mathrm{~mm}$, rotation speed $0.5 \mathrm{~s}$, and helical pitch $1.0875: 1$ or $1.375: 1$. For pregnant women: tube voltage $120 \mathrm{kV}$, automatic tube current (10-300) $\mathrm{mA}$, thickness (7-10) $\mathrm{mm}$, slice interval $5 \mathrm{~mm}$, rotation speed $0.6 \mathrm{~s}$, helical pitch 1.375:1, and noise index 15. Thyroid, abdomen, and plevis were protected by the lead sheath. The dose-length product (DLP) was 50-150 mGy.cm. For children: tube voltage $100 \mathrm{kV}$, automatic tube current (30-100) $\mathrm{mA}$, thickness (3-5) $\mathrm{mm}$, slice interval $1 \mathrm{~mm}$, rotation speed $0.6 \mathrm{~s}$, and helical pitch 0.969:1. Lung window images at 0.625 to $1 \mathrm{~mm}$ thickness were reconstructed. Iterative reconstruction technique was implemented. The informed consents for CT examination were obtained from all patients.

\section{Image interpretation}

The chest CT features of non-pregnant adults were reviewed by two radiologists (HL and JL, with 8 and 10 years of experience in thoracic imaging, respectively) in consensus. The chest CT features of pregnant women and children were reviewed by two radiologists ( $\mathrm{HL}$ and $\mathrm{FL}$, with 8 and 15 years of experience in thoracic imaging, respectively) in consensus. The disputes between the radiologists were resolved by consultation with another third experienced radiologist (DW or WL, with more than 25 years of experience in thoracic imaging, respectively).

CT features consisted of lesion number, distribution, as well as presence of ground-glass opacity (GGO), GGO with consolidation, GGO with reticulation from intralobular/interlobular septal thickening, consolidation, air bronchogram, cavitation, lymphadenopathy, and pleural effusion. GGO was defined as a hazy density increase in lung with vessels margins preservation, whereas consolidation was opacification with obscuring vessels margins. ${ }^{19}$ The distribution included focal (one lesion either in unilateral or bilateral lungs), scattered (less than 10 lesions either in unilateral or bilateral lungs), and diffuse patterns (more than 10 lesions in bilateral lungs) and bilaterality and unilaterality which divided into the peripheral (the outer one-third of the lung) and central locations. Regarding the number of lesions, the lesion was counted as the involved lung segments if a lesion extends across multiple lung segments. ${ }^{20}$ Lymphadenopathy was defined as the size of lymph node more than $10 \mathrm{~mm}$ in short-axis diameter.

In order to stratify the degree of lung involvement, a scoring method based on CT findings was implemented. Each of the lung lobe involvement was classified as none (0\%), minimal (1\%-25\%), mild $(26 \%-50 \%)$, moderate $(51 \%-75 \%)$, or severe $(76 \%-100 \%)$, the corresponding scores were $0,1,2,3$, and 4 , respectively. The final "total severity score" (TSS) was obtained by summing the scores of the five lobes. ${ }^{21}$

\section{Statistical analysis}

Statistical analysis was performed with SPSS 23.0 (IBM, New York, NY) and $P<0.05$ indicated a statistically significant difference. Quantitative variables were expressed as mean (standard deviation, SD). The one-way analysis of variance (ANOVA) and the 
H. Liu, F. Liu and J. Li et al./Journal of Infection $x x x(x x x x) x x x$

Table 1

Clinical and laboratory characteristics of 55 adult patients with COVID-19 pneumonia.

\begin{tabular}{|c|c|c|}
\hline Characteristics & Number (\%)* & $P$ value* \\
\hline \multicolumn{3}{|l|}{ Gender } \\
\hline Male & $5(36 \%) / 0(0 \%) / 0(0 \%)$ & \\
\hline Female & $9(64 \%) / 16(100 \%) / 25(100 \%)$ & \\
\hline Age(years) & $33.5(27-58) / 30(26-35) / 31(22-42)$ & 0.001 \\
\hline Wuhan area & $11(79 \%) / 16(100 \%) / 25(100 \%)$ &.. \\
\hline \multicolumn{3}{|l|}{ Initial Symptoms } \\
\hline Fever & $14(100 \%) / 7(44 \%) / 9(36 \%)$ & $<0.001$ \\
\hline Post-partum fever & $0(0 \%) / 5(31 \%) / 9(36 \%)$ & 0.038 \\
\hline Cough & $9(64 \%) / 6(38 \%) / 9(36 \%)$ & 0.195 \\
\hline Short of breath & $0(0 \%) / 2(13 \%) / 3(12 \%)$ & 0.390 \\
\hline Fatigue & $2(14 \%) / 3(19 \%) / 2(8 \%)$ & 0.590 \\
\hline Loss of appetite & $0(0 \%) / 2(13 \%) / 1(4 \%)$ & 0.294 \\
\hline Diarrhea & $1(7 \%) / 0(0 \%) / 0(0 \%)$ & 0.225 \\
\hline \multicolumn{3}{|l|}{ Laboratory test } \\
\hline Leukocytosis & $0(0 \%) / 8(50 \%) / 9(36 \%)$ & 0.010 \\
\hline Leukopenia or normal & $14(100 \%) / 8(50 \%) / 16(64 \%)$ & .. \\
\hline Elevated neutrophil ratio & $2(14 \%) / 14(88 \%) / 20(80 \%)$ & $<0.001$ \\
\hline Lymphopenia & $11(79 \%) / 9(56 \%) / 16(64 \%)$ & 0.430 \\
\hline Decreased lymphocyte ratio & $12(86 \%) / 16(100 \%) / 22(88 \%)$ & 0.315 \\
\hline Elevated C-reactive protein & $7(50 \%) / 13(81 \%) / 14(56 \%)$ & 0.154 \\
\hline
\end{tabular}

* Data are shown with the percentage in parentheses except for the age with range in the parentheses, and the data are demonstrated in the order of non-pregnant adults group, laboratory-confirmed pregnant women group, and clinically-diagnosed pregnant women group.

** Differences of clinical characteristics among the non-pregnant adults, laboratory-confirmed pregnant women, and clinically-diagnosed pregnant women groups.

Pearson Chi-squared test were used for the comparison of continuous data and categorical data among the non-pregnant adult group and the two pregnant groups.

\section{Role of the funding source}

There was no funding source for this study.

\section{Results}

Clinical findings

Clinical findings of 55 adult patients were shown in Table 1. Among the 41 pregnant women, the median age was 30 years old (range, 22-42 years). The gestational age ranged from 22 weeks to 40 weeks plus 5 days. Nineteen patients were admitted to hospital for delivery and 22 were outpatients. And four had gestational diabetes, 3 gestational hypertension, and 1 hepatitis B infection. Of them, 16 patients were laboratory-confirmed SARS-CoV-2 positive, 17 laboratory-negative but with typical CT features of COVID-19 pneumonia and the other 8 did not undergo SARS-CoV-2 test. Among the laboratory negative patients, SARS-CoV-2 RNA was tested three times in 2 patients, twice in 1 patient, and once in the other patients. The populations without SARS-CoV-2 positive were categorized into the clinically-diagnosed group. There were $9 / 16(56 \%)$ and $16 / 25$ (64\%) patients presenting initial normal body temperature in the laboratory-confirmed and clinicallydiagnosed pregnant groups, which was less common than the nonpregnant adults group $(P<0.001)$. Totally 6 and 10 patients in the laboratory-confirmed and clinically-diagnosed pregnant groups underwent delivery, in which 5 and 9 patients presented post-partum low grade fever before CT examination. No significant differences existed for the other clinical symptoms in our study. All the pregnant women with COVID-19 were stratified as the mild or common types. No pregnant woman was admitted to the intensive care unit (ICU), and no clinical abnormalities were observed in the infants.

The leukocytosis (50\% and 36\%) and elevated neutrophil ratio ( $88 \%$ and $80 \%$ ) were more common in the laboratory-confirmed and clinically-diagnosed pregnant groups $(P=0.010, P<0.001)$ compared with the non-pregnant adult group ( $0 \%$ and $14 \%)$, while there was no significant difference for lymphopenia among the three groups.

As with the 14 non-pregnant adults, the most common symptoms were also fever (100\%) and cough (64\%). The clinical characteristics of the 4 children were shown in Table 2, the blood test was non-specific. Two of them were sister and brother. One child was simultaneously with respiratory syncytial virus (RSV) and SARS-CoV-2 positive, while all the other adults and children were tested negative for mycoplasma pneumonia, chlamydia pneumonia, respiratory syncytial virus, adenovirus, coxsackie virus $B$, influenza $\mathrm{A}$, influenza $\mathrm{B}$, and human parainfluenza virus.

\section{Chest CT features}

The initial CT features were demonstrated in Table 3. The median interval of CT scan from the onset of symptoms was 2 days (range, 1-8 days). Sixteen pregnant women underwent CT scans after delivery and the others prior to delivery. A total of 614 lesions were detected in the 55 adult patients. The lesions involved 174 lobes and 390 lung segments. There were 299 lesions (49\%) located in the lower lobes and 488 (79\%) located peripherally (Fig. 1). The most common lesions were pure GGO, GGO with consolidation, GGO with reticulation, and complete consolidation.

Both the non-pregnant and the pregnant groups shared the same peripheral distribution of the lesions in the lungs (Figs. 2 and 3) without significant difference $(P=0.241)$. However, consolidation including mixed GGO with consolidation and complete consolidation was significantly more frequent in the laboratoryconfirmed and clinically-diagnosed pregnant groups compared to the non-pregnant adults $(P=0.007, P<0.001)$, while GGO and GGO with reticulation were more frequent in the non-pregnant adults $(P=0.001, P<0.001)$. No significant difference was found regarding consolidation between the two pregnant groups $(P=0.366)$. Focal and scattered distributions were presented in $0(0 \%) / 9$ (56\%), 1 (7\%)/5 (31\%), and $6(43 \%) / 9$ (36\%) patients in the non-pregnant adult, laboratory-confirmed pregnant, and clinicallydiagnosed pregnant groups, respectively. Diffuse distribution was detected in 5 (36\%), 7 (44\%), and $10(40 \%)$ patients in the above three groups, respectively. Then the mean involved lungs segments were 3.4 (SD 1.4), 3.0 (SD 2.0), and 3.1 (SD 1.8) in the three 
H. Liu, F. Liu and J. Li et al./Journal of Infection $x x x(x x x x) x x x$

Table 2

Clinical and chest CT imaging characteristics of 4 children with laboratory-confirmed COVID-19 pneumonia.

\begin{tabular}{ll}
\hline Findings & Case $1 /$ Case2/Case3/Case 4 \\
\hline Clinical characteristics & \\
Gender & \\
$\quad$ Male/Female & Female/Male/Female/Male \\
Age & 5 years/11 months/9 years/2 months \\
Exposure to confirmed or suspected patient & Yes/Yes/Yes/Yes \\
Initial Symptoms & Yes/Yes/Yes/No \\
Fever & Yes/Yes/No/Yes \\
Cough & Yes/No/No/No \\
Fatigue & No/No/No/Yes \\
Other pathogen infection & \\
Laboratory test & Decreased/Normal/Normal/Normal \\
Blood leukocyte count & Decreased/Decreased/Normal/Normal \\
Neutrophil ratio & Increased/Increased/Normal/Normal \\
Lymphocyte count & Increased/Increased/Decreased/Normal \\
Lymphocyte ratio & Normal/Normal/Normal/Increased \\
C-reactive protein & Normal/Single consolidation/Single pure GGO/Multiple consolidations \\
CT findings & \\
Other findings & No/No/No/Yes \\
Pleural effusion & No/No/No/No \\
Lymphadenopathy & \\
\hline
\end{tabular}

GGO, ground-glass opacity.

Table 3

Chest CT imaging characteristics of 55 adult patients with COVID-19 pneumonia.

\begin{tabular}{|c|c|c|c|}
\hline Characteristics & Number (\%)* & Lesion (\%)* & $P$ value \\
\hline \multicolumn{4}{|l|}{ Location } \\
\hline No lesion & $0(0 \%) / 3(19 \%) / 0(0 \%)$ & 0 &.. \\
\hline Unilateral lung & $3(21 \%) / 3(19 \%) / 9(36 \%)$ & $10(8 \%) / 7(4 \%) / 26(8 \%)$ & 0.303 \\
\hline Bilateral lungs & $11(79 \%) / 10(63 \%) / 16(64 \%)$ & $121(92 \%) / 154(96 \%) / 296(92 \%)$ &.. \\
\hline \multicolumn{4}{|l|}{ Lung lobe involvement } \\
\hline No lesion & $0(0 \%) / 3(19 \%) / 0(0 \%)$ & 0 & .. \\
\hline One lobe & $1(7 \%) / 2(13 \%) / 8(32 \%)$ & $3(2 \%) / 2(1 \%) / 14(4 \%)$ & 0.149 \\
\hline Two lobes & $3(21 \%) / 1(6 \%) / 2(8 \%)$ & $10(8 \%) / 5(3 \%) / 24(7 \%)$ & 0.144 \\
\hline Three lobes & $4(29 \%) / 2(13 \%) / 4(16 \%)$ & $33(25 \%) / 16(10 \%) / 27(8 \%)$ & $<0.001$ \\
\hline Four lobes & $1(7 \%) / 2(13 \%) / 1(4 \%)$ & $4(3 \%) / 23(14 \%) / 9(3 \%)$ & $<0.001$ \\
\hline Five lobes & $5(36 \%) / 6(38 \%) / 10(40 \%)$ & $81(62 \%) / 115(71 \%) / 248(77 \%)$ & 0.004 \\
\hline \multicolumn{4}{|l|}{ Predominant distribution } \\
\hline Peripheral & $14(100 \%) / 16(100 \%) / 24(96 \%)$ & $100(76 \%) / 135$ (84\%) / $253(79 \%)$ & 0.241 \\
\hline Central & $0(0 \%) / 0(0 \%) / 1(4 \%)$ & $31(24 \%) / 26(16 \%) / 69(21 \%)$ &.. \\
\hline \multicolumn{4}{|l|}{ Main presence of lesions } \\
\hline Pure GGO & $13(93 \%) / 12(75 \%) / 18(72 \%)$ & $94(72 \%) / 91(57 \%) / 168(52 \%)$ & 0.001 \\
\hline GGO with consolidation & $11(79 \%) / 7(44 \%) / 15(60 \%)$ & $25(19 \%) / 30(19 \%) / 95(30 \%)$ & 0.009 \\
\hline GGO with reticulation & $10(71 \%) / 6(38 \%) / 4(16 \%)$ & $24(18 \%) / 9(26 \%) / 16(5 \%)$ & $<0.001$ \\
\hline Consolidation & $3(21 \%) / 8(50 \%) / 14(56 \%)$ & $12(9 \%) / 40(25 \%) / 58(18 \%)$ & 0.002 \\
\hline \multicolumn{4}{|l|}{ Other findings } \\
\hline Pleural effusion & $1(7 \%) / 6(38 \%) / 6(24 \%)$ & 0 & .. \\
\hline Lymphadenopathy & $0(0 \%) / 0(0 \%) / 0(0 \%)$ & 0 & .. \\
\hline
\end{tabular}

GGO, ground-glass opacity.

* Data are shown with the percentage in parentheses and the data are demonstrated in the order of non-pregnant adults group, laboratory-confirmed pregnant women group, and clinically-diagnosed pregnant women group.

** Differences of Chest CT imaging characteristics for lesions among the non-pregnant adults, laboratory-confirmed pregnant women, and clinically-diagnosed pregnant women groups.

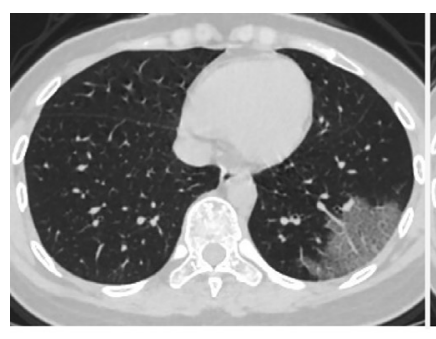

a

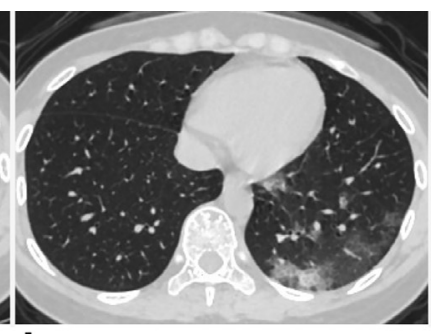

b

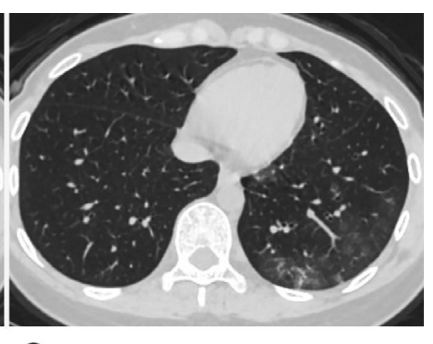

C

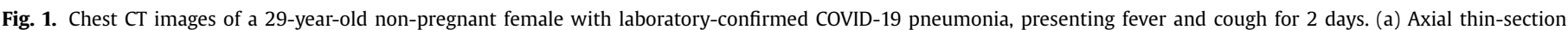

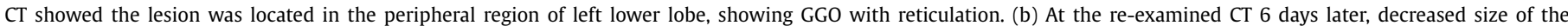

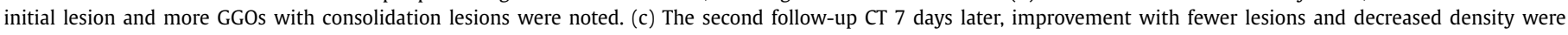
depicted. 


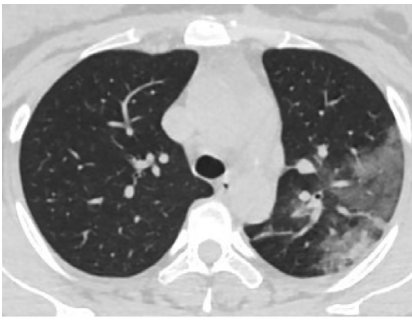

a

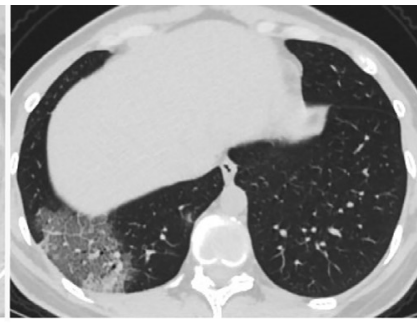

b

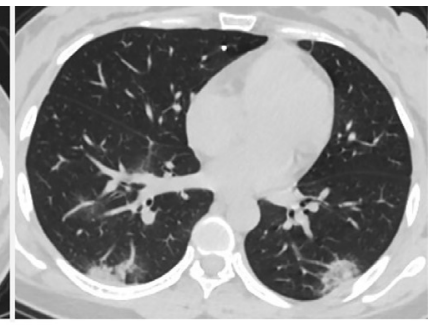

C

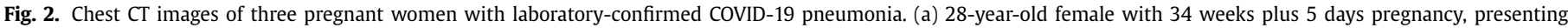

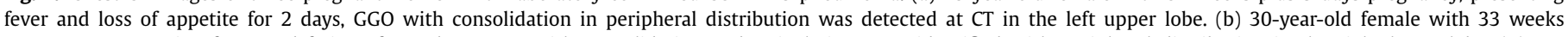

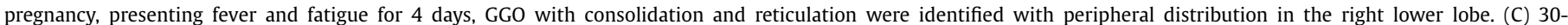

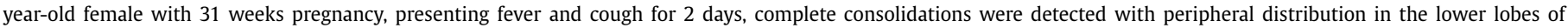
bilateral lungs.

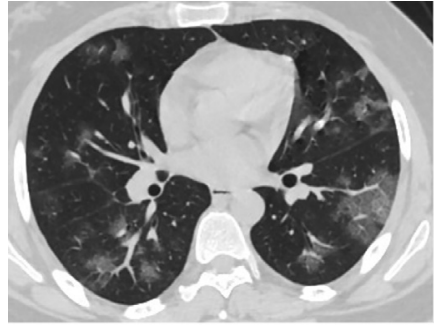

a

Fig. 3. Chest CT images of a 28-year-old female with 36 weeks plus 1 day pregnancy, with clinically-diagnosed COVID-19 pneumonia, presented post-partum low grade fever. (a) Baseline CT imaging demonstrated typical CT features of COVID-19 pneumonia, with multiple GGOs and GGOs with reticulation lesions predominantly peripheral distribution in both lungs. (b) Follow-up CT 10 days later after antiviral therapy, demonstrated improvement with fewer lesions and few consolidation lesions left.

groups, while the mean involved pulmonary segments were 6.8 (SD 5.2), 6.3 (SD 4.9), and 7.8(SD 6.4). The TSS tended to be higher in the clinically-diagnosed group than that in the laboratoryconfirmed group (4.8 [SD 3.5] vs. 4.4 [SD3.3]). The TSS in the non-pregnant adult group was 4.3 (SD 2.4), and there were no significant differences among the three groups $(P=0.882)$. In the laboratory-confirmed pregnant group, no pulmonary abnormalities associated with COVID-19 were found in three patients with post-partum fever, and CT just revealed mild pulmonary exudation and pleural effusion which could be referred to as the common post-partum findings. GGO with reticulation was detected in $10 / 14(71 \%), 6 / 16(38 \%)$, and $4 / 25(16 \%)$ patients among the nonpregnant, laboratory-confirmed, and clinically-diagnosed pregnant groups $(P<0.001)$, while $3 / 14(21 \%), 7 / 16(44 \%)$, and $9 / 25(36 \%)$ for the air bronchogram.

The Chest CT findings of the children were also shown in Table 2. One 2-month-old child with simultaneous RSV and SARSCoV-2 infections demonstrated multiple focal consolidation lesions and pleural effusion (Fig. 4).

\section{Follow-up}

After antiviral therapy, 6 patients in the non-pregnant adult groups, 6 in the laboratory-confirmed pregnant group, and 8 patients in the clinically-diagnosed pregnant group, had follow-up CT. The time intervals from the initial CT ranged from 3 to 15 days. Nine patients (9/13) demonstrated mild progression at reexamined CT after 3 days, and 6 patients (6/8) demonstrated improvement 6-9 days after the initial CT scans. Four patients with progressive changes were performed with serial follow-up CT later till 13 days or 15 days since first CT. Three children had follow-up
CT. No new lesion was found 9 days later in the child with normal initial CT, and her brother demonstrated improvement at CT on the same day. Another child showed improvement at the follow-up CT after 7 days.

\section{Discussion}

In the present study, we focused on investigating the clinical and chest CT imaging characteristics of the COVID-19 pneumonia in pregnant women and children, besides those from the non-pregnant adults. Our study revealed that the clinical symptoms of pregnant women were atypical in comparison with the non-pregnant adults. Leukocytosis (41\%) and elevated neutrophil ratio (83\%) were unusually noted for the COVID-19 cases. The consolidation lesions were more prevalent in the pregnant cases. The cases in the clinically-diagnosed pregnant group were vulnerable to more pulmonary involvement at CT in which disputes for aggravating the patients' status took place due to the possible false negative or postponed coronavirus nucleic acid tests. Therefore, it is commonly speculated that some delay in management was worrisome in order to wait for a confirmed diagnosis. For limiting the epidemic, chest CT has been playing an indispensable role in selection of the candidates for quarantine in Wuhan and other places in China. For children, the CT findings were diverse from normal appearances to a few lesions including GGO or consolidation in lungs.

The ongoing outbreak COVID-19 pneumonia demonstrated a relatively high contagion; meanwhile there are no specific therapeutic drugs and vaccines for the COVID-19. Early identification of COVID-19 is important for the patients as well as the healthy population. Currently, the patients should be isolated for treatment from the healthy people for controlling the epidemic. It has been reported that chest CT is superior to RT-PCR in sensitivity for early detection of COVID-19.22 Based on the epidemiological characteristics, clinically-diagnosed cases with typical CT features were treated with the same therapy in Hubei Province to offset the effects of possible false negative of the new coronavirus nucleic acid tests. ${ }^{13}$ In our study, $44 \%$ and $36 \%$ pregnant women presented initial onset of fever in either laboratory-confirmed or clinicallydiagnosed group, which was less common than $87.9 \%$ in the nonpregnant population in the study by Guan et al. ${ }^{11}$ and consistent with the previous study enrolling 9 pregnant women with COVID19. ${ }^{17}$ Currently, fever served as the basic screening flag in China. Absence of typical symptoms increased the difficulty of the surveillance in the pregnant population. Furthermore, the physiological findings about leukocytosis and elevated neutrophil ratio due to adaptations to gestation could further complicate the situation. Nevertheless, no pregnant women were admitted to ICU. Compared with the previous studies, ${ }^{11,20}$ the patients' median age was much younger (30 years old) with fewer comorbidities in our study, thus 


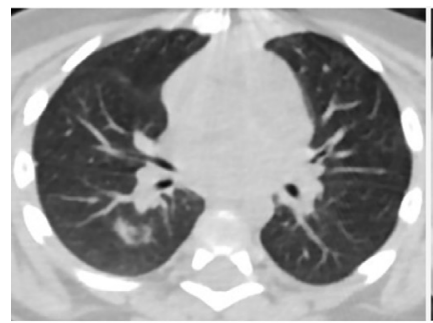

a

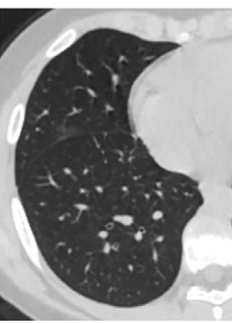

b

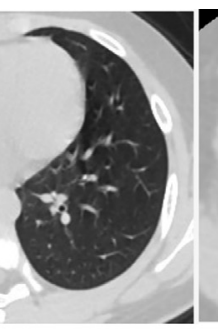

C

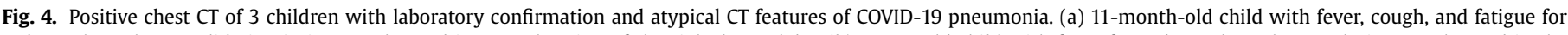

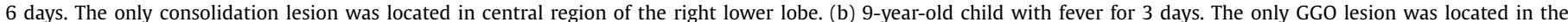

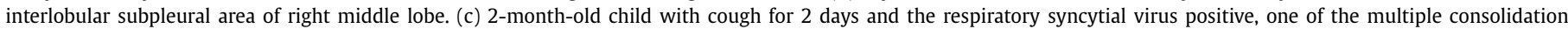
lesions with air bronchogram was located in the right upper lobe, and pleural effusion was also noted.

it may be one of the reasons there were more cases of pregnant women categorized as mild or common type in clinical context.

As with unenhanced chest CT imaging of pregnant women during the epidemic in Wuhan, low dose technique was implemented with the DLP ranging from 50 to $150 \mathrm{mGy} \mathrm{cm}$ for diagnostic imaging in our study, which was safe for the fetuses. ${ }^{23}$ Sixteen pregnant women were performed with post-partum CT in our study. As with the CT features associated with COVID-19 pneumonia, the common characteristics in the pregnant group included pure GGO, GGO with consolidation or reticulation, and complete consolidation with predominantly peripheral distribution and bilateral lung involvement, which were similar to those of the non-pregnant group in our research as reported in the previous studies. ${ }^{19,20,24}$ There were overlaps in imaging findings between COVID-19 and other viral infections, such as the coronavirus SARS-CoV and MERS-CoV pneumonia, as well as H1N1, H5N1, and H7N9 infections. ${ }^{19,20}$ The patterns of GGOs or GGO with the above features in predominantly peripheral distribution will facilitate the diagnosis of COVID-19 pneumonia. ${ }^{20}$ The above imaging findings could be mostly explained by a recently published research on the pathological investigation of COVID-19. ${ }^{25}$ Diffuse alveolar injury superimposed with cellular fibromyxoid exudates and hyaline membrane formation was identified on pathology. Other findings included pulmonary edema and interstitial mononuclear inflammatory infiltrates (dominated by lymphocytes). Therefore, we have the GGOs, consolidations, and reticulations which indicate fibromyxoid exudates, the interstitial edema and the lymphocytes infiltrate resulting in the lymphopenia inside the blood. The more common consolidation and relatively higher TSS in the laboratoryconfirmed and clinically-diagnosed pregnant women suggested the more severity in pregnant women, except for three laboratoryconfirmed pregnant women showing no abnormalities related with COVID-19 pneumonia at CT. Therefore, there is no doubt that the CT study did a big favor in structuring the clinical treatment strategy for the pregnant women as we hoped. Fortunately, the infants were all normal without any evidence of vertical transmission from women with COVID-19 pneumonia in late pregnancy, which was consistent with the prior research by Chen et al. ${ }^{16}$

While compared between the two pregnant groups, the consolidation were more frequent in the clinically-diagnosed group with a relatively higher TSS of 4.8 (SD 3.5), indicating relatively more severe lung involvement resulting from delayed treatment prior to a laboratory confirmation. Cases with initial negative RT-PCR for SARS-CoV-2 but high clinical suspicion were also reported in previous studies, and the SARS-CoV-2 was positive after repeated tests. $^{12,13}$ For the clinically-diagnosed patients, it is speculated that samples from the lower respiratory tract via lavage and repeat tests may be helpful to rule out the false negative results. Fortunately, the laboratory-negative cases with typical imaging findings were supposed to receive the same treatment protocol as the positive ones in China. Pleural effusion was identified in 6/16 (38\%) and 6/25 (24\%) patients in the laboratory-confirmed and clinically-diagnosed pregnant groups, which was more than that in the study by Song et al. ${ }^{20}$ This phenomenon could be attributed to a common finding within 1-24 h of normal delivery. ${ }^{26}$

Compared with adults, fewer children are infected with SARSCoV-2. Nine children younger than 14 years out of 1011 patients were reported by Guan et al. ${ }^{11}$ Our study revealed that the non-specific abnormalities (pure GGO or consolidation) distributed without peripheral predominance at $\mathrm{CT}$ unlike the adults. However, when superimposed with other pathogen infections, the pulmonary involvement was more severe, as simultaneous infection of RSV and SARS-CoV-2 in one child was detected in our study. Nevertheless, it was difficult to distinguish if the consolidation was associated with COVID-19 pneumonia, since the consolidation and pleural effusion were also common in pure RSV infection. ${ }^{27}$ The contact history and clinical symptoms of fever or cough may be helpful for the surveillance.

We performed the follow-up CT studies 3 days and 7 days after the initial CT. Compared with the previous studies, ${ }^{24,28}$ most of the cases in our study demonstrated earlier progressive changes in lungs at first follow-up CT at 3 days. And most of them were witnessed to get improvements at second follow-up CT at about 7 days (6-9 days). Therefore, CT could be regarded as an efficient tool for the early detection and monitoring the dynamic evolution in the patients with COVID-19. ${ }^{24}$

Our study has several limitations. Firstly, the sample size was relatively small. Larger population from more centers should be warranted to validate our preliminary results. In addition, even though it's a study conducted in two centers, the majority of patients was from the hospital majoring in women and children, then the selection bias existed. Secondly, 22 pregnant women are outpatients, thus the follow-up CT images were absent for monitoring the therapeutic effects. Finally, for the pregnant woman and children, low dose technique was implemented so that the image quality was not so good as that of the non-pregnant adult group, but fortunately it's sufficient to evaluate the lesions.

In conclusion, our preliminary study demonstrated that the clinical findings of pregnant women with COVID-19 pneumonia were atypical, bringing about difficulties in early detection. The clinically-diagnosed pregnant cases shared similar chest CT features with the laboratory-confirmed ones, but with more severe consolidation lesions, and repeated SARS-CoV-2 tests should be implemented for these highly clinical suspicious patients, however, the negative tests will not hinder the management based on the typical clinical and imaging findings in clinical context where the COVID-19 is life-threatening. Chest CT study was the modality of choice for early detection, severity assessment, and monitoring the therapeutic effects with or without SARS-CoV-2 confirmation for this population. The chest CT imaging features of children with 
COVID-19 pneumonia were non-specific, while the exposure history and clinical symptoms could be more helpful for the screening.

\section{Contributors}

HL, FL, DW, and WL conceived the study conception and design. $\mathrm{HL}$ and FL contributed to the literature search. HL, FL, JL, and TZ performed the acquisition of data. HL and JL conducted the analysis and interpretation of data. HL and FL contributed to the figures. HL, FL and JL drafted the manuscript. DW and WL contributed to critical revision of the manuscript.

\section{Declaration of Competing Interest}

We declare no competing interests.

\section{References}

1. Zhu N, Zhang D, Wang W, Li X, Yang B, Song J, et al. A Novel Coronavirus from Patients with Pneumonia in China, 2019. N Engl J Med 2020;382(8):727-33.

2. Huang C, Wang Y, Li X, Ren L, Zhao J, Hu Y, et al. Clinical features of patients infected with 2019 novel coronavirus in Wuhan. China. Lancet 2020;395(10223):497-506

3. Chen N, Zhou M, Dong X, Qu J, Gong F, Han Y, et al. Epidemiological and clinical characteristics of 99 cases of 2019 novel coronavirus pneumonia in Wuhan, China: a descriptive study. Lancet 2020;395(10223):507-13.

4. The International Committee on Taxonomy of Viruses (ICTV) Coronaviridae Study Group. Naming the 2019 Coronavirus. https://talk.ictvonline.org/.

5. Chan JF, Yuan S, Kok KH, To KK, Chu H, Yang J, et al. A familial cluster of pneumonia associated with the 2019 novel coronavirus indicating person-to-person transmission: a study of a family cluster. 2020;395(10223):514-523.

6. Phan LT, Nguyen TV, Luong QC, Nguyen TV, Nguyen HT, Le HQ, et al. Importation and Human-to-Human Transmission of a Novel Coronavirus in Vietnam. $N$ Engl J Med 2020;382(9):872-4.

7. Wang D, Hu B, Hu C, Zhu F, Liu X, Zhang J, et al. Clinical Characteristics of 138 Hospitalized Patients With 2019 Novel Coronavirus-Infected Pneumonia in Wuhan, China. JAMA 2020 Feb 7 Epub ahead of print. doi:10.1001/jama.2020. 1585.

8. National Health Commission of China. "Report of the latest situation of novel coronavirus pneumonia in China as of February 18, 2020". http://www.nhc.gov. cn/xcs/yqtb/202002/8f2cfd17f4c040d89c69a4b29e99748c.shtml .

9. Yang Y, Lu Q, Liu M, Wang Y, Zhang A, Jalali N, et al. Epidemiological and clinical features of the 2019 novel coronavirus outbreak in China. medRxiv. 2020;2020 02.10.20021675.

10. Li Q, Guan X, Wu P, Wang X, Zhou L, Tong Y, et al. Early Transmission Dynamics in Wuhan, China, of Novel Coronavirus-Infected Pneumonia. N Engl J Med 2020 Jan 29 Epub ahead of print. doi:10.1056/NEJMoa2001316.
11. Guan WJ, Ni ZY, Hu Y, Liang WH, Ou CQ, He JX, et al. Clinical characteristics of 2019 novel coronavirus infection in China. medRxiv. 2020;2020 02.06.20020974.

12. Xie X, Zhong Z, Zhao W, Zheng C, Wang F, Liu J. Chest CT for Typical 2019nCoV Pneumonia: Relationship to Negative RT-PCR Testing. Radiology 2020 Feb 12 200343. Epub ahead of print. doi:10.1148/radiol.2020200343.

13. Huang P, Liu T, Huang L, Liu H, Lei M, Xu W, et al. Use of Chest CT in Combination with Negative RT-PCR Assay for the 2019 Novel Coronavirus but High Clinical Suspicion. Radiology 2020;295(1):22-3.

14. National Health Commission of China. "Diagnosis and Treatment for COVID-19 Pneumonia (Fifth Trial Version)". http://www.nhc.gov.cn/.

15. Mor G, Cardenas I, Abrahams V, Guller S. Inflammation and pregnancy: the role of the immune system at the implantation site. Ann N Y Acad Sci 2011;1221:80-7.

16. Chen H, Guo J, Wang C, Luo F, Yu X, Zhang W, et al. Clinical characteristics and intrauterine vertical transmission potential of COVID-19 infection in nine pregnant women: a retrospective review of medical records. The Lancet 2020;395(10226):809-15.

17. Zhu H, Wang L, Fang C, Peng S, Zhang L, Chang G, et al. Clinical analysis of 10 neonates born to mothers with 2019-nCoV pneumonia. Translational Pediatrics 2020;9(1):51-60.

18. World Health Organization. Technical-guidance for 2019-nCoV RT-PCR https://www.who.int/emergencies/diseases/novel-coronavirus-2019/technicalguidance/laboratory-guidance

19. Chung M, Bernheim A, Mei X, Zhang N, Huang M, Zeng X, et al. CT Imaging Features of 2019 Novel Coronavirus (2019-nCoV). Radiology 2020;295(1):202-7.

20. Song F, Shi N, Shan F, Zhang Z, Shen J, Lu H, et al. Emerging Coronavirus 2019-nCoV Pneumonia. Radiology 2020;295(1):210-17.

21. Chang YC, Yu CJ, Chang SC, Galvin JR, Liu HM, Hsiao CH, et al. Pulmonary sequelae in convalescent patients after severe acute respiratory syndrome: evaluation with thin-section CT. Radiology 2005;236(3):1067-75.

22. Ai T, Yang Z, Hou H, Zhan C, Chen $C$, Lv W, et al Correlation of Chest $\mathrm{CT}$ and RT-PCR Testing in Coronavirus Disease 2019 (COVID-19) in China: A Report of 1014 Cases. Radiology 2020 Feb 26 200642. Epub ahead of print. doi:10.1148/ radiol.2020200642.

23. Toma P, Bartoloni A, Salerno S, Granata C, Cannata V, Magistrelli A, et al. Protecting sensitive patient groups from imaging using ionizing radiation: effects during pregnancy, in fetal life and childhood. Radiol Med 2019;124(8):736-44.

24. Shi $\mathrm{H}$, Han $\mathrm{X}$, Jiang $\mathrm{N}, \mathrm{Cao} \mathrm{Y}$, Alwalid $\mathrm{O}, \mathrm{Gu}$ J, et al. Radiological findings from 81 patients with COVID-19 pneumonia in Wuhan, China: a descriptive study The Lancet Infectious Diseases 2020 Feb 24 pii: S1473-3099(20)30086-4. Epub ahead of print. doi:10.1016/S1473-3099(20)30086-4.

25. Xu Z, Shi L, Wang Y, Zhang J, Huang L, Zhang C, et al. Pathological findings of COVID-19 associated with acute respiratory distress syndrome. Lancet Respir Med 2020 Feb 18 pii: S2213-2600(20)30076-X. Epub ahead of print. doi:10.1016/ S2213-2600(20)30076-X

26. Gourgoulianis KI, Karantanas AH, Diminikou G, Molyvdas PA. Benign postpartum pleural effusion. Eur Respir J 1995;8(10):1748-50.

27. Koo HJ, Lim S, Choe J, Choi SH, Sung H, Do KH. Radiographic and CT Features of Viral Pneumonia. Radiographics 2018;38(3):719-39.

28. Pan F, Ye T, Sun P, Gui S, Liang B, Li L, et al. Time Course of Lung Changes On Chest CT During Recovery From 2019 Novel Coronavirus (COVID-19) Pneumonia. Radiology 2020 Feb 13 200370. Epub ahead of print. doi:10.1148/radiol. 2020200370. 